\title{
A New Inexpensive Simulation Model for Ultrasound Assessment of Optic Nerve Sheath Diameter
}

\author{
Garrett G. R. J. Johnson (1D, Tomislav Jelic, Angela Derksen, Bertram Unger, \\ Frederick Zeiler ${ }^{(D)}$ Markus Ziesmann, Lawrence M. Gillman
}

\begin{abstract}
Background: Sonographic measurement of optic nerve sheath diameter (ONSD) is becoming increasingly accepted as a diagnostic modality to detect elevations in intracranial pressure. As this technique becomes more widespread, methods to address the inherent operator-dependent nature of this modality will need to be developed. We propose a novel low-cost model to accurately simulate sonographic ONSD measurement for purposes of training and assessment. Methods: We designed models composed of medical tubing of various diameters readily available from typical hospital supplies and suspended them in gelatin. The models were evaluated by ultrasound by three expert point-of-care sonographers using a standard linear array probe and technique proposed in the literature. Results: This model generates faithful simulation of the ONS that closely approximates in vivo images and can be used to produce accurate, reproducible measurements. Materials are low cost and easy to acquire and assemble. Conclusions: Our model provides realistic simulated images of the ONS. Through comparison of sonographic measurements to the known tube diameters, this model serves as a promising inexpensive tool to teach the method of ultrasound assessment of ONSD or as a way to determine accuracy of this novel ultrasound technology.

RÉSUMÉ : Nouveau modèle de stimulation peu coûteux pour évaluer le diamètre de la gaine du nerf optique à l'aide d'ultrasons. Contexte : La mesure échographique du diamètre de la gaine du nerf optique est une modalité de diagnostic de plus en plus acceptée afin de détecter des élévations de la pression intracrânienne. Au fur et à mesure que cette technique se répand, il sera nécessaire de développer des méthodes permettant de tenir compte du fait que cette modalité de diagnostic dépend intrinsèquement de celui ou celle qui l'administre. À cet égard, nous voulons proposer ici un nouveau modèle pour simuler avec précision et à faible coût la mesure échographique du diamètre de la gaine du nerf optique, et ce, à des fins de formation et d'évaluation. Méthodes : C'est partir de fournitures hospitalières typiques que nous avons conçu des modèles de tubes médicaux de différents diamètres facilement accessibles. Pour ce faire, nous les avons suspendus préalablement dans la gélatine. Nos modèles ont été ensuite évalués à l'aide d'ultrasons par trois échographistes spécialisés disponibles dans des lieux de soins. Ils ont eu recours à une sonde standard à réseau linéaire (standard linear array probe) et se sont basés sur une technique proposée dans la littérature scientifique. Résultats : Nos modèles ont produit des simulations fidèles de la mesure échographique du diamètre de la gaine du nerf optique, s'approchant dans les faits étroitement des images in vivo. On a aussi pu les utiliser pour obtenir des mesures précises et reproductibles. Ajoutons enfin que le matériel utilisé, en plus d'être bon marché, est facile à acquérir et à assembler. Conclusions : Nos modèles fournissent en somme des images simulées réalistes de la mesure échographique du diamètre de la gaine du nerf optique. Grâce à des comparaisons effectuées avec les mesures échographiques des diamètres connus de tubes, ces modèles constituent des outils prometteurs et peu coûteux pour enseigner la méthode d'évaluation du diamètre de la gaine du nerf optique par ultrasons ou bien encore une façon de déterminer la précision de cette nouvelle technologie par ultrasons.
\end{abstract}

Keywords: Handheld ultrasound, Intracranial pressure, Optic nerve sheath diameter, Point-of-care ultrasound, Ultrasound doi:10.1017/cjn.2020.37

Can J Neurol Sci. 2020; 47: 543-548

\section{INTRODUCTION}

Elevated intracranial pressure (ICP) is a serious condition in neurocritically ill patients. ${ }^{1}$ If not diagnosed and treated promptly, it has been shown to lead to secondary brain injury due to decreased cerebral perfusion pressure. ${ }^{2,3}$ Invasive intracranial monitoring is currently the gold standard for diagnosis of

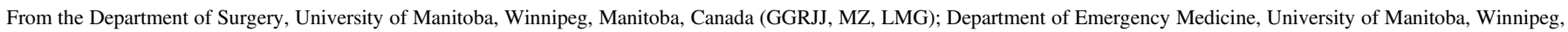

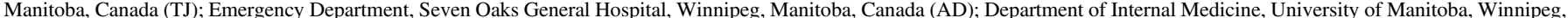

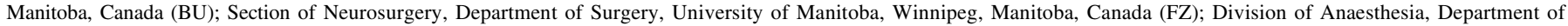
Medicine, University of Cambridge, Cambridge, UK (FZ)

Received August 1, 2019. Final Revisions Submitted January 14, 2020. Date of Acceptance February 13, 2020.

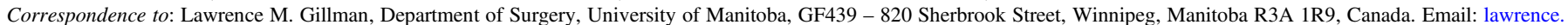
gillman@umanitoba.ca 

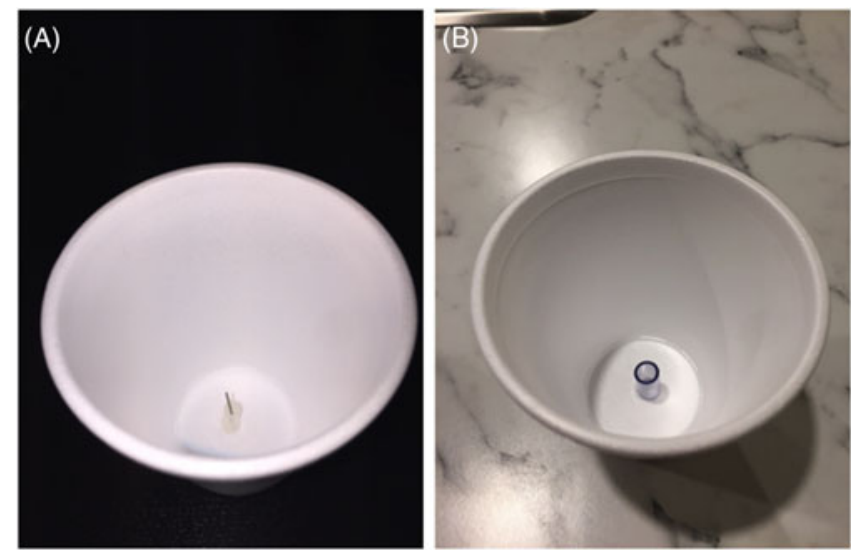

Figure 1: Model making process. (A) Intravenous tubing held upright by pin and glued to the base of a coffee cup. (B) Nasogastric tube after pin is removed held upright by dried glue alone.

intracranial hypertension ${ }^{4}$; however, it can be expensive, requires neurosurgical expertise, and is associated with a risk of bleeding and infection. ${ }^{5}$

Sonographic assessment of optic nerve sheath diameter (ONSD) as a non-invasive means to assess ICP has become increasingly popular in the literature. ${ }^{6}$ Acute increases in ICP have been shown to strongly correlate with increases in ONSD. ${ }^{7-10}$ Recently, ultrasound measurement of ONSD has been investigated for use as a dynamic real-time ICP monitoring tool in critically ill patients with promising results. ${ }^{10}$ Ultrasound itself is becoming increasingly available due to decreasing costs, the production of portable pocket ultrasound systems, and the dissemination of ultrasound expertise. As access to ultrasound spreads, sonographic assessment of ONSD will continue to have increasing uptake as a diagnostic test. Ultrasound is particularly useful in less well-equipped and austere environments where other tools such as invasive monitors and neurosurgical expertise, CT scanners, or MRI are not readily available, but where rapid assessment of ICP is still essential. Furthermore, ultrasound can be used as an adjunct to the physical exam in order to help justify the use of more expensive tests. As with any ultrasound measurement, ONSD sonography has been shown to be operator dependent with small to moderate inter-observer and intra-observer variation reported in the literature. ${ }^{1-16}$ It has been previously shown that this variance can be improved with practice. ${ }^{15,17}$

There is growing evidence that simulation is effective for developing and assessing both technical and non-technical skills. ${ }^{18}$ It allows for practice in areas of weakness, rarity, critical importance, or procedures that are uncomfortable to the patient. However, simulation can be very elaborate or cost prohibitive, thus defeating its purpose of increasing opportunities to practice otherwise inaccessible skills. Previously, we have described a standardized model using a gelatin globe with a 3D printed disc to generate a simulated ONS of consistent dimensions in order to test inter- and intra-observer variability. ${ }^{12,14,19}$ However, as this measurement technique relied on a shadow generated by the disc that may be distinct from the size of the disc itself, we did not have an adequately reliable physical parameter to measure, in order to directly compare our sonographic ONS measurements. Here, we describe the design and development of a new model to
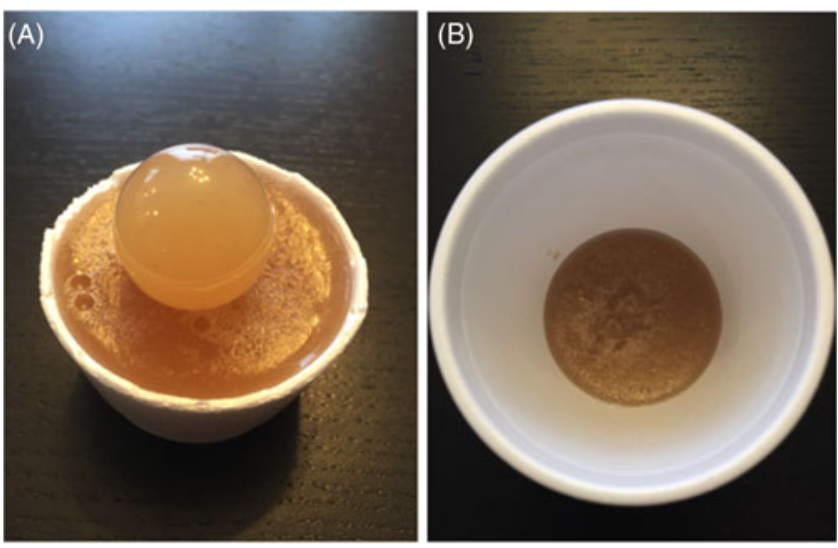

Figure 2: Finished models of tubes suspended in gelatin and psyllium (A) with optional gelatin eyeball and (B) without eyeball.

simulate the ONS using cost-effective tubes and demonstrate that it can be used to acquire ultrasound images that can be measured accurately and are similar in appearance to those acquired from in vivo sources. This model can be used not only as a low-cost instructional tool to teach the method of ONSD measurement but also has the potential for use as a research tool for determining accuracy in measurement of predetermined ONS sizes in a reproducible and controlled setting.

\section{Materials And Methods}

ONS models were constructed as an improvement to previously described methods. ${ }^{19-21}$ They are made from: $237 \mathrm{ml}(8 \mathrm{oz})$ white disposable coffee cups (Dart Container Company, Mason, MI, USA), white glue (Elmer's School Glue, Elmer Products, Inc. Highport, NC, USA), straight pins, unflavored gelatin (Knox-brand, E.D. Smith Foods, Ltd., Winona, ON, Canada), sugar-free psyllium powder (Metamucil Sugar Free Dietary Fiber Supplement, Procter \& Gamble, Cincinnati, OH, USA), and assorted sizings of synthetic polymer medical tubing. We used four different types of tubing in seven diameters obtained from excess/expired material in our intensive care unit to simulate the ONS: a $6 \mathrm{~mm}$ disposable portex tracheostomy inner cannula (Smiths Medical, Minneapolis, MN, USA), $4.2 \mathrm{~mm}$ sheath from a spring-wire guide (Arrow brand, Teleflex Inc., Wayne, PA, USA), $3.5 \mathrm{~mm}$ polyvinyl IV tubing (B. Braun Medical Inc., Englewood, NJ, USA), and various sizes of gastric sump tubes (Salem sump tubes, Medtronic, Dublin, Ireland). Tubing was selected for their varying diameters simulating the range of ONSD found in nature (3-8 $\mathrm{mm})$.

First, to simulate the ONS, the medical tubing was cut into a $1-\mathrm{cm}$ long segment and placed with the long axis perpendicular to the base of the coffee cup. A small pin was placed through the bottom of the cup vertically through the center of the tubing in order to keep it in place. Next, the bottom end of the tubing was fixed to the cup base with a generous portion of glue and allowed to dry for an hour with the pin in place to keep the tubing upright. After the glue had dried, the pin was removed from the base of the coffee cup with the tubing now suspended upright by the glued bottom (Figure 1). The resultant pinpoint hole in the bottom of the cup was covered externally with a piece of tape. Using a variation of a previously described recipe for gelatin ultrasound phantoms, ${ }^{20}$ we suspended three packets of gelatin in $100 \mathrm{ml}$ of 


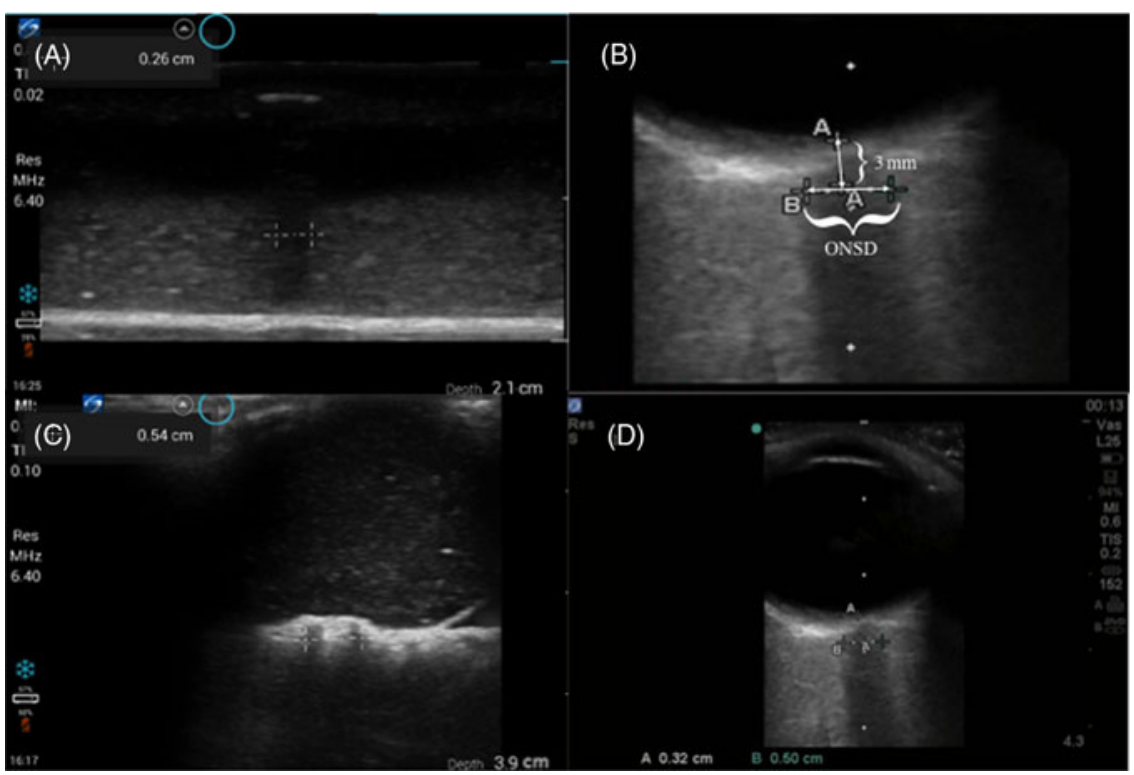

Figure 3: Ultrasound images of model optic nerve sheaths (left) compared to in vivo human eye (right). (A) Simulation model made from $3.5 \mathrm{~mm}$ polyvinyl intravenous tube without gelatin globe, $(B)$ in vivo human eye close-up of optic nerve with measurement technique highlighted. Optic nerve sheath diameter is taken $3 \mathrm{~mm}$ behind the globe. (C) 16 French nasogastric tube model with gelatin globe, $(D)$ entire in vivo image of $5 \mathrm{~mm}$ human ONS.

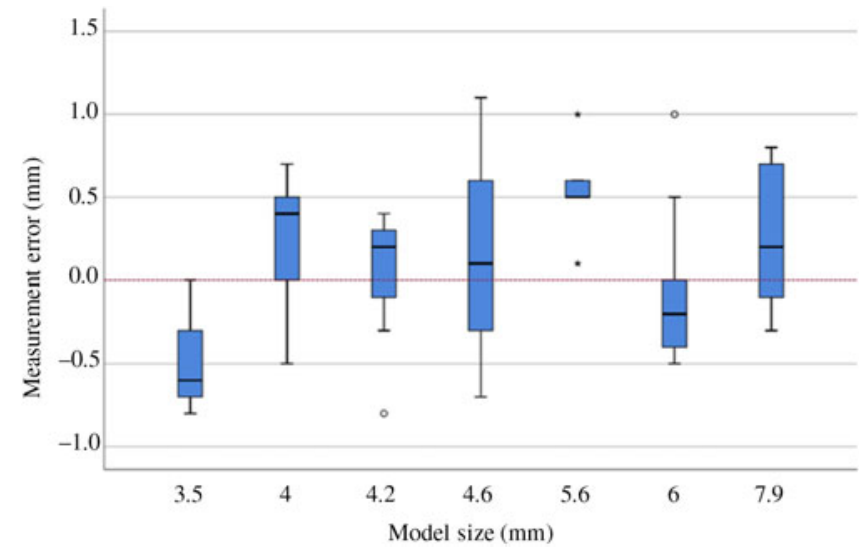

Figure 4: Box and whisker plot of measurement error (observed minus predicted) stratified by predicted size of model optic nerve sheath diameter as measured by digital calipers. $o=$ outlier, $*=$ extreme outlier.

room-temperature water. We brought a separate $150 \mathrm{ml}$ to a boil and gradually mixed in the gelatin suspension with the boiling water over medium heat until the gelatin was completely dissolved (1-2 min). Next, one tablespoon of psyllium powder was added and whisked slowly until it completely dissolved ( 1 min). Using this method reduces bubbles or clumping of the gelatin compared to Bude and Adler's originally described method where the gelatin packets were added directly to the boiling water. ${ }^{20}$ Finally, the hot mixture was poured slowly into the coffee cups to a few millimeters above the upper level of the tubing and placed in the refrigerator for at least $1 \mathrm{~h}$ to allow the gelatin to congeal. Models could be stored in sealed sandwich bags or covered plastic containers in a refrigerator at $4{ }^{\circ} \mathrm{C}$ for up to 3 weeks before desiccating or becoming moldy.

As an optional step in order to simulate the optic globe above the ONS, the molten gelatin mixture can be poured into a 1-inch spherical baking mold (Chicago School of Mold Making, Oak Park, IL, USA) as previously described. ${ }^{19}$ A semi-circular defect can then be carved into the hardened gelatin above the tubing in the coffee cups, lubricated with a generous portion of ultrasound gel, and the gelatin balls placed in the defect (Figure 2).

The models were evaluated utilizing a $13-6 \mathrm{MHz}$ linear array ultrasound transducer (L25x transducer, Sonosite Corp., Bothell, WA, USA) and a portable ultrasound (Sonosite M-Turbo, SonoSite Inc., Bothell, WA, USA) using the ophthalmic manufacturer preset. Measurement of ONSD was conducted using the "black-stripe" method described in the literature. This is performed by placement of the probe over the orbit and identification of the ONS as a black line behind the globe. This black line is the optic nerve, and its diameter is measured $3 \mathrm{~mm}$ behind the optic disc (Figure 3). ${ }^{7-9,22,23}$ Measurements were conducted by three expert point-of-care ultrasonographers (LG, TJ, AD) in a single session. The diameters were $3.5 \mathrm{~mm}$ (polyvinyl intravenous line tubing), $4 \mathrm{~mm}$ (nasogastric tube), $4.2 \mathrm{~mm}$ (spring wire guide sheath), $4.6 \mathrm{~mm}$ (nasogastric tube), $5.5 \mathrm{~mm}$ (nasogastric tube), $6 \mathrm{~mm}$ (tracheostomy cannula), and $7.9 \mathrm{~mm}$ (nasogastric tube). Sonographers measured each model in random order a total of three times. Operators were blinded to the identity of the model, to the actual diameters of the tubing, and to each other's measurements.

All statistical analyses were performed using SPSS Statistics version 25 (IMB, Armonk, NY, USA). The measurements obtained by the ultrasound devices were compared to the physical diameter of the tubes as measured with digital calipers by a separate investigator (GJ) and their measurement error was calculated.

\section{Results}

Sonographic images of our models shown next to an in vivo image of ONSD from a live person are shown in Figure 3. Measurement error (observed by ultrasound minus predicted by 
caliper measurement) for each model size is summarized in Figure 4. The mean error across all measurements is $0.10 \mathrm{~mm}$ and the error variance is $0.26 \mathrm{~mm}^{2}$

\section{Discussion}

Our simulation model for sonographic ONSD measurement provides a low-cost realistic opportunity for trainees to practice this relatively difficult task without discomfort to a patient. The images obtained of the tubing by ultrasound can be measured and provide realistic simulation of the ONS (Figure 3). Furthermore, these measurements can be made with a low degree of error (Figure 4), similar to those previously published. ${ }^{12}$

Simulation of the orbit and its surrounding structures has been described in the literature in various models in the past. To our knowledge, there are only two models that simulate the ONS. ${ }^{19,21}$ In a previous study, our group used a gelatin globe with a 3D-printed disc that generated a sonographic "shadow" to simulate the ONS. While this produced good visual results, and the disc could be created in any desired dimensions, the shadow was not necessarily the same size as the disc itself and served as a poor standard. ${ }^{19}$ Printed discs required access to a specialized 3D printer, which is cost prohibitive, limiting the utility of this model as a low-cost training aid. Our current model addresses both of these deficiencies. Medical tubing was used, as excess is readily salvageable from hospital supplies (e.g., expired, opened, used, etc.) at low cost. The diameter of the simulated ONSD is a direct measurement of the tube reflection itself, not its ultrasound shadow, so there is an objectively verified diameter for comparison. The tubes come in a variety of sizes relevant to ONSD measurement.

More recently, Murphy et al. used a water-filled ping-pong ball and vinyl tubing submerged in gelatin to simulate the orbit and ONS. While this method produced excellent still images that were indistinguishable from in vivo images by emergency medicine residents, it had a number of limitations. Very low gain was needed in order to minimize reverberation artifact from the ping-pong balls. ${ }^{21}$ This was inconsequential when study personnel obtained still photographs. However, if this model were to be used as a training aid where novices would be expected to attain the images themselves, this could decrease fidelity of the simulation. While not difficult to learn, ${ }^{15,17}$ we believe that a significant challenge of sonographic determination of ONSD is acquiring the best image quality. Image acquisition skills must be demonstrated and practiced along with image interpretation skills. Moreover, using a gelatin globe instead is inexpensive, easier to produce, and has less reverberation artifact than the ping-pong ball method. For even clearer images, psyllium can be removed from the recipe as previously described, ${ }^{19}$ and construction of a spherical globe can be abandoned, allowing one to practice measuring the diameter of a tube suspended in gelatin alone.

Murphy et al. also used polyvinyl tubing to simulate the ONS. ${ }^{21}$ Search from various manufacturers however shows the smallest diameter readily available is approximately $6 \mathrm{~mm}$, which only simulates the upper end of possibilities of ONSD found in vivo. Medical tubing such as that used in our model comes in a large range of diameters across the normal and abnormal ONS measurement spectrum.

Further benefits to our model as an educational tool include the potential ability to assess learners' progression as they develop the skill of ONSD measurement. The diameter of the tubing used in our models can be preselected to simulate a variety of normal or pathologic ONSD. The actual physical diameter of the tubing is measured rather than a shadow; thus, an instructor can know the actual diameter of the ONS by pre-measuring it in advance in order to more objectively assess a learner's ability to determine the diameter. Previously, agreement with expert sonographer, ${ }^{15}$ agreement with other imaging modalities, ${ }^{24,25}$ or a decrease in intra-observer variation over time ${ }^{17}$ would be the only ways to monitor learner progression of ONSD measurement.

Although performing ocular ultrasound on humans is safe, ${ }^{26}$ phantom models such as ours are convenient, easily accessible, and allow for prolonged scan time without risk of discomfort or injury to the retina. This could be particularly important for novice sonographers who may be developing their skills in image acquisition and adjusting device settings. Furthermore, practice on a low-cost model can allow trainees to perform dedicated practice of this technique on their own time, which may be important for skills acquisition and maintenance given strict duty hour restrictions imposed on trainees in many jurisdictions. ${ }^{27}$ In addition, having an ONSD of a known size allows for an evaluation of competence and accuracy of trainee measurements. While volunteers can be free and even more realistic, they are often in short supply and could be reserved for those trainees that have already gained some degree of competence on a practice model.

Although standard portable ultrasound units have been extensively tested and have been determined to be reasonably accurate for measurement of ONSD and determination of ICP, ${ }^{11-13,15,16}$ newer handheld units are continuously being added to the market. New pocket ultrasound devices are not identical to previously validated portable ultrasound, nor are they identical to the pocket Vscan unit that we have previously found to be accurate. ${ }^{14}$ These ultrasound units can have small screen sizes, different probe frequencies, employ new touch screen technology and software presets than machines that have been previously validated for, and are currently not approved for ocular use. ${ }^{28-30}$ These differences can be reasonably assumed to affect how accurately an operator can measure a subcentimeter structure such as the optic nerve sheath. Our simulation model with known reproducible sizes of ONSD could be an invaluable tool for testing novel ultrasound units as they hit the market. This could provide manufacturers with the rationale to apply for and implement the required safety presets onto their pocket devices so that they could be used for this use in the future. Sonographic assessment of simulated ONSD can be compared for agreement with actual tube diameter to determine the accuracy of measurement of new units as they become available.

Despite the expected benefits of our novel model, there are some limitations and areas for future improvement. Most notably, our model can only simulate the popular "black stripe" method for measurement of OSND. Recently, new criteria have been proposed to improve image quality of sonographic measurement of ONSD and standardize measurements across different observers and scans. ${ }^{31}$ Early data suggest that these criteria may lead to a change in normal values for ONSD measurement. ${ }^{9}$ These criteria emphasize bilateral ocular sonography and avoidance or identification of landmarks within and around the globe, which are not simulated in our model. To our knowledge, no model to date has been developed which is able to simulate all structures necessary to apply the new quality criteria. 
Our model in its current state lasts approximately 3 weeks if kept in a sealed container in a refrigerator. If kept for longer, or improperly stored, it will desiccate rapidly or become colonized with fungus. Alternative materials such as agarose, ballistic gelatin, silicone, polyacrylamide, polyvinyl alcohol, polyurethane, oil gel, animal and human cadaveric tissue, and others have all been studied, ${ }^{32}$ but none are accessible by a simple trip to the grocery store (in the case of knox gelatin and psyllium) and many are more expensive and require specialized equipment or a laboratory environment to make. Further study on easily accessible preservatives that can be added to the gelatin without affecting its sonographic properties or user safety in order to extend the life of the model would be helpful, though preservation may ultimately not be important with affordable and easily reproduced models.

\section{Conclusions}

Our simulation model for sonographic ONSD measurement provides a low-cost option to practice this relatively difficult task. The images obtained of the tubing by ultrasound can be measured accurately, with low error, and provided realistic simulation of the ONS for purposes of training or further research.

\section{Ethics Approval and Consent to Participate}

This study was deemed not to require research ethics board review by the University of Manitoba Health Research Ethics Board.

\section{Statement of Authorship}

All authors were involved in study design, data analysis, and manuscript composition. LMG, GGRJJ, TJ, and AD were involved in data collection. All authors read and approved the final manuscript.

\section{Availability of Data and Materials}

The datasets used and/or analyzed during the current study are available from the corresponding author on reasonable request.

\section{FUNDING}

FZ's research program is supported through the University of Manitoba Thorlakson's Chair in Surgical Research Establishment Grant, University of Manitoba VPRI Research Investment Fund (RIF), Winnipeg Health Sciences Center Foundation, and the University of Manitoba Rudy Falk Clinician-Scientist Professorship.

BU's research is funded in part by unrestricted grant from advanced bionics which is being administered in conjunction with Mitacs.

This research was supported by a General Operating Grant from the Health Science Centre Foundation and a GFT Research Grant from the Department of Surgery, University of Manitoba, both in Winnipeg, Manitoba, Canada.

\section{DiscLOSURES}

The authors have no conflicts of interest to declare.

\section{ReFERENCES}

1. Pickard JD, Czosnyka M. Management of raised intracranial pressure. J Neurol, Neurosurg Psychiatry. 1993;56(8):845-58.

2. Schreiber MA. Determinants of mortality in patients with severe blunt head injury. Arch Surg. 2002;137(3):285.

3. Badri S, Chen J, Barber J, et al. Mortality and long-term functional outcome associated with intracranial pressure after traumatic brain injury. Intensive Care Med. 2012;38(11):1800-9.

4. Carney N, Totten AM, O'Reilly C, et al. Guidelines for the management of severe traumatic brain injury. Neurosurgery. 2016:1-244.

5. Ragland J, Lee K. Critical care management and monitoring of intracranial pressure. J Neurocrit Care. 2016;9(2):105-12.

6. Robba C, Santori G, Czosnyka M, et al. Optic nerve sheath diameter measured sonographically as non-invasive estimator of intracranial pressure: a systematic review and meta-analysis. Intensive Care Med. 2018;44(8):1284-94.

7. Geeraerts T, Merceron S, Benhamou D, Vigué B, Duranteau J. Non-invasive assessment of intracranial pressure using ocular sonography in neurocritical care patients. Intensive Care Med. 2008;34(11):2062-7.

8. Kimberly HH, Shah S, Marill K, Noble V. Correlation of optic nerve sheath diameter with direct measurement of intracranial pressure. Acad Emerg Med. 2008;15(2):201-4.

9. Soliman I, Johnson GGRJ, Gillman LM, et al. New optic nerve sonography quality criteria in the diagnostic evaluation of traumatic brain injury. Crit Care Res Pract. 2018;2018:1-7.

10. Chen L-M, Wang L-J, Hu Y, Jiang X-H, Wang Y-Z, Xing Y-Q. Ultrasonic measurement of optic nerve sheath diameter: a noninvasive surrogate approach for dynamic, real-time evaluation of intracranial pressure. Br J Ophthalmol. 2018. Available at: https://bjo.bmj.com/content/early/2018/10/25/bjophthalmol-2018312934.long\#ref-8.

11. Ballantyne SA, O’Neill G, Hamilton R, Hollman AS. Observer variation in the sonographic measurement of optic nerve sheath diameter in normal adults. Eur J Ultrasound. 2002;15(3):145-9.

12. Zeiler FA, Unger B, Zhu Q, et al. A unique model for ONSD part II: inter/intra-operator variability. Can J Neurol Sci. 2014;41(4): $430-5$.

13. Bäuerle J, Lochner P, Kaps M, Nedelmann M. Intra- and interobserver reliability of sonographic assessment of the optic nerve sheath diameter in healthy adults. J Neuroimaging. 2012;22(1): $42-5$.

14. Johnson GGRJ, Zeiler FA, Unger B, Hansen G, Karakitsos D, Gillman LM. Estimating the accuracy of optic nerve sheath diameter measurement using a pocket-sized, handheld ultrasound on a simulation model. Crit Ultrasound J. 2016;8(1):845.

15. Potgieter DW, Kippin A, Ngu F, McKean C. Can accurate ultrasonographic measurement of the optic nerve sheath diameter (a non-invasive measure of intracranial pressure) be taught to novice operators in a single training session? Anaesth Intensive Care. 2011;39:95-100.

16. Oberfoell S, Murphy D, French A, Trent S, Richards D. Inter-rater reliability of sonographic optic nerve sheath diameter measurements by emergency medicine physicians. J Ultrasound Med. 2017;36(8):1579-84.

17. Zeiler FA, Ziesmann M, Goeres P, et al. A unique method for estimating the reliability learning curve of optic nerve sheath diameter ultrasound measurement. Crit Ultrasound J. 2016;8(1):4.

18. Munshi F, Lababidi H, Alyousef S. Low- versus high-fidelity simulations in teaching and assessing clinical skills. J Taibah Univ Med Sci. 2015;10(1):12-5.

19. Zeiler FA, Unger B, Kramer AH, Kirkpatrick AW, Gillman LM. A unique model for ultrasound assessment of optic nerve sheath diameter. Can J Neurol Sci. 2013;40(2):225-9.

20. Bude RO, Adler RS. An easily made, low-cost, tissue-like ultrasound phantom material. J Clin Ultrasound. 1995;23(4):271-3.

21. Murphy DL, Oberfoell SH, Trent SA, French AJ, Kim DJ, Richards DB. Validation of a low-cost optic nerve sheath ultrasound phantom: an educational tool. J Med Ultrasound. 2017;25: 96-100. 
22. Geeraerts T, Launey Y, Martin L, et al. Ultrasonography of the optic nerve sheath may be useful for detecting raised intracranial pressure after severe brain injury. Intensive Care Med. 2007; 33(10): 1704-11.

23. Blaivas M, Theodoro D, Sierzenski PR. Elevated intracranial pressure detected by bedside emergency ultrasonography of the optic nerve sheath. Acad Emerg Med. 2003;10(4):376-81.

24. Ohle R, McIsaac SM, Woo MY, Perry JJ. Sonography of the optic nerve sheath diameter for detection of raised intracranial pressure compared to computed tomography: a systematic review and meta-analysis. J Ultrasound Med. 2015;34(7):1285-94.

25. Bäuerle J, Schuchardt F, Schroeder L, Egger K, Weigel M, Harloff A. Reproducibility and accuracy of optic nerve sheath diameter assessment using ultrasound compared to magnetic resonance imaging. BMC Neurol. 2013;13(1):2014.
26. Shankar H, Pagel PS. Potential adverse ultrasound-related biological effects: a critical review. Anesthesiology. 2011;115(5):1109-24.

27. Nataraja RM, Webb N, Lopez P-J. Simulation in paediatric urology and surgery. Part 1: an overview of educational theory. J Pediatr Urol. 2018;14(2):120-4.

28. Lumify Ultrasound System User Manual. Philips Healthcare; 2016.

29. Sonosite IVIZ User Manual. FUJIFILM SonoSite, Inc.; 2018.

30. Butterfly $\mathrm{iQ}^{\mathrm{TM}}$ personal ultrasound system user manual. Butterfly Network, Inc.; 2019.

31. Sargsyan AE, Blaivas M, Geeraerts T, Karakitsos D. Ocular ultrasound in the intensive care unit. In: Lumb P, Karakitsos D, editors. Critical Care Ultrasound; 2014.

32. Culjat MO, Goldenberg D, Tewari P, Singh RS. A review of tissue substitutes for ultrasound imaging. Ultrasound Med Biol. 2010;36(6):861-73. 